\title{
Smart Grid Coordination of a Chemical Processing Plant
}

\author{
Jianyuan Feng $^{(1)}$, Avery Brown ${ }^{(1)}$, Dennis O’Brien ${ }^{(2)}$ and Donald J. Chmielewski ${ }^{(1)}{ }_{*}$ \\ ${ }^{(1)}$ Department of Chemical and Biological Engineering, Illinois Institute of Technology, Chicago, IL \\ (2) Jacobs Consultancy, Chicago, IL \\ * Corresponding author (312-567-3537, chmielewski@iit.edu)
}

As the electric power grid evolves to a smart grid, the introduction of real-time price structures will provide consumers with the opportunity to obtain low cost (possibly negative cost) electrical energy. This work will propose a novel design for a utility plant within a chemical processing facility that will enable exploitation of the diurnal nature of expected electricity prices. Specifically, we will investigate an oil heating, gas fired furnace that has been augmented with an electric heater. A second configuration, that augments the electric heater with an energy storage unit, will also be investigated. Results indicate that substantial savings in energy cost can be achieved, but will likely be undercut by the capital costs associated with the electric heater.

\section{INTRODUCTION}

The operation of grid scale power systems is transitioning from a centralized single party approach to a deregulated (market based) approach that encourages active participation from multiple stakeholders, $[1,2,8]$. Under such schemes the cost of electric energy is expected to track 
consumer demand. If electric energy consumption is low, then only low cost generators (coal and nuclear) need be online. Conversely, when consumer demand is high, higher cost generators (combined cycle gas turbines) will need to be brought online. At no point can there be an imbalance between consumption and generation, [3]. If such an event were to occur, there would be a catastrophic failure on the grid, likely leading to a blackout. The net result is a price of electricity that tracks the diurnal cycle of consumer demand (see Figure 1).

In parallel there has been substantial growth in renewable power sources (wind and solar). However, the intermittent nature of these sources is expected to increase the volatility of electricity prices, especially if dispatch capable fossil plants are decommissioned, [5]. If renewable power is available and demand is low, then prices could drop below the operational costs of the base-load plants (coal and nuclear) - notice the negative price during the second day of Figure 1. On the other hand, if renewable power is not available while consumer demand is high, prices may spike due to the required use of very high cost generators (simple cycle gas turbines, also known as peaker plants).

The notion of Demand Response (DR) envisions consumers participating in the operation of the grid, $[6,7]$. While there are many DR mechanisms, [8], the current effort will focus on economic response. Under such a program, consumers will be incentivized by the price of electricity to use more or less electric energy at particular times of the day. A number of efforts have advocated the use of DR in a residential setting $[9,10,31]$, as well as in commercial buildings $[11,12,13,14,33,34]$. In the industrial sector, a number of DR opportunities have been explored, including; aluminum smelting, [15], steel production, [16], industrial gas production and 
distribution, [17, 18], and chemical processing [19, 20].

The current effort focuses on a chemical processing facility in the sense that the energy used to drive a portion of the process is delivered via a hot oil utility stream, serviced by a natural gas fired furnace. The proposition is to augment this furnace with an electric heater, as illustrated in Figure 2. As we will see, optimal operation of this augmented system can be achieved using a policy that is a function of fuel and electricity prices, but is independent of time. This policy is akin to that resulting from a real-time optimization algorithm.

It should be highlighted that many chemical processing facilities purchase electric energy based on market prices through their energy management system. However, these energy management systems rarely use energy prices to influence the operation of a chemical plant. The current effort proposes one possibility of allowing energy prices to influence plant operation, but the scope is restricted to the utility plant and does not attempt to make any changes to the production side of the plant. While changes to production schedules, based on electric energy prices, may yield a theoretical economic benefit [20], the practical impact on equipment degradation and safety procedures is unknown and will result in a level of risk that most in the chemical industry are unwilling to accept.

In many cases, use of an energy storage device will enhance the performance of a smart grid coordinated system $[14,20,21,32]$. As such, we will also investigate the configuration of Figure 3, which employs a molten salt energy storage unit along with a secondary heat exchange operation. In addition to an increase in the number of decision variables, at both the design and operational levels, the need to account for the time history of the storage device will require the use of 
Economic Model Predictive Control (EMPC). General descriptions of EMPC can be found in [22, 23, 24], while application of EMPC to smart grid coordination problems can be found in [11, 12, $13,14,17,20,21,25,33,34]$.

The choice to used thermal energy as the storage medium, as opposed to electrochemical batteries or gas compression, warrants some discussion. In the proposed configuration the end use of the stored energy is to heat a fluid. Thus, the most natural storage medium is thermal, since only one energy conversion step is needed - electric to thermal. If one were to use some other storage medium, then several conversion steps would be needed - for example a battery requires electric to chemical, chemical to electrical and finally electric to thermal. Since each of these conversion steps will have losses, the option with fewer steps is expected to be most efficient. A similar approach was used in [32], where compressed air was selected as the storage medium because the end use was to augment the compressed air stream into an air separation unit.

\section{ANALYSIS OF THE ELECTRIC HEATER ONLY CASE}

The envisioned furnace will heat an oil utility stream using natural gas as the energy source. We assume that the required rate of heat to the oil is constant for all time and equal to $P_{\text {load }}$. If the conversion efficiency of the furnace is $\eta_{f}$, then the required amount of fuel is $P_{\text {load }} / \eta_{f}$. Since the heat load is fixed, it is reasonable to define this as the maximum fuel rate to the furnace: $P_{f}^{\max }=$ $P_{\text {load }} / \eta_{f}$. The operating cost for this baseline case is calculated as $\sum_{i=1}^{M} c_{f}(i) P_{f}^{\max }$ where $c_{f}(i)$ is the cost of fuel (in $\$ / M M B T U$ or equivalently in $\$ / M W h$ ) during time interval $i$, and $M$ is the number of time intervals of the analysis. 
As indicated in Figure 2, the electric heater augmented system will have the following energy balance:

$$
\eta_{f} P_{f}(i)+\eta_{e} P_{e}(i)=P_{\text {load }}
$$

where $P_{e}(i)$ is the electric power sent to the heater during time interval $i$, and $\eta_{e}$ is the conversion efficiency of the heater. Notice that the heater has been placed upstream of the furnace, so that the existing oil temperature regulation equipment can continue to be employed. However, doing so carries the implicit assumption that the furnace will remain online at all times. Thus, we assume a maximum furnace turndown factor of $\delta$, indicating that $P_{f}^{\min }=(1-\delta) P_{f}^{\max }$. This limit could equivalently be stated in terms of power limits to the electric heater

$$
0 \leq P_{e}(i) \leq P_{e}^{\max } \hat{=}\left(P_{\text {load }}-\eta_{f} P_{f}^{\min }\right) / \eta_{e}
$$

Given the configuration of Figure 2, the question is to determine the appropriate values for $P_{f}(i)$ and $P_{e}(i)$ during each hour $i$. This will be done by solving the following optimization problem:

$$
\Phi=\min _{P_{f}(i), P_{e}(i)}\left\{\sum_{i=1}^{M} c_{e}(i) P_{e}(i)+c_{f}(i) P_{f}(i)\right\} \quad \text { s.t. } \quad(1),(2) i=1 \ldots M
$$

where $c_{e}(i)$ is the cost of electric energy during period $i$. Due to the time separability of this problem, an equivalent solution can be found by solving the following optimization problem at each time interval $i$ :

$$
\phi_{i}=\min _{P_{f}(i), P_{e}(i)}\left\{c_{e}(i) P_{e}(i)+c_{f}(i) P_{f}(i)\right\} \quad \text { s.t. } \quad(1),(2) \quad \text { and } \quad \Phi=\sum_{i=1}^{M} \phi_{i}
$$

Problem (4) can be further simplified by substituting (1) into the objective function to yield:

$$
\phi_{i}=\min _{P_{e}(i)}\left\{c_{R}(i) P_{e}(i)\right\}+c_{f}(i) P_{\text {load }} / \eta_{f} \quad \text { s.t. } \quad 0 \leq P_{e}(i) \leq P_{e}^{\max }
$$


where $c_{R}(i)=c_{e}(i)-c_{f}(i) \eta_{e} / \eta_{f}$. In this form, the solution to Problem (4) is easily concluded to be:

$$
\begin{aligned}
& P_{e}^{*}(i)=\left\{\begin{array}{clc}
0 & \text { if } & c_{R}(i) \geq 0 \\
P_{e}^{\max } & \text { if } & c_{R}(i)<0
\end{array}\right. \\
& \phi_{i}=\left\{\begin{array}{cll}
c_{f}(i) P_{\text {load }} / \eta_{f} & \text { if } & c_{R}(i) \geq 0 \\
c_{e}(i) P_{e}^{\max }+c_{f}(i) P_{f}^{\min } & \text { if } & c_{R}(i)<0
\end{array}\right.
\end{aligned}
$$

Example 1: Assume $P_{\text {load }}=300 \mathrm{MMBTU} / \mathrm{hr}=87.9 \mathrm{MW}, \eta_{f}=0.75, \eta_{e}=0.98$ and $\delta=39 \%$. Then, $P_{f}^{\min }=71.5 \mathrm{MW}$ and $P_{e}^{\max }=35 \mathrm{MW}$. Furthermore, consider the electricity and rescaled natural gas prices of Figure 4, $[4,26]$. The rescaling is a result of multiplying natural gas prices by $\eta_{e} / \eta_{f}$. The convenience of this rescaling is that if the rescaled gas price is higher than electricity price then $c_{R}(i)<0$ and $P_{e}{ }^{*}(i)=35 \mathrm{MW}$, otherwise $c_{R}(i) \geq 0$ and $P_{e}{ }^{*}(i)=0$. Such behavior is illustrated in the plots of Figure 5. The top plot shows significant utilization of the electric heater, while the bottom plot shows much less utilization. However, in the calculation of operating cost savings, the amount of time the heater is used is less important than the price differential $c_{R}(i)$, when $c_{R}(i)<0$. The top plot of Figure 6 illustrates numerous periods where the cost of electricity is significantly lower than fuel costs. Conversely, the bottom plot of Figure 6 shows very small price differentials and thus one should expect little change in operating costs even though the optimization calls for wide and frequent swings in operating conditions. Similar conclusions can be drawn from the annual plots of Figure 4 and are supported by the quantitative results of Table 1 . In each case $M=365 \times 24$ hours.

Based on the limited information of Figure 4, we observe that average electricity price is 
correlated with natural gas prices (see Figure 7). However, we also note that the variablity of electricity prices also seems to track gas prices (see Figure 7). This is likely due to the fact that the daily peaks in electricity price result from the operation of gas turbine based electric generation during periods of high electric power demand. Conversely, during periods of low power demand only coal and nuclear based plants are in operation, which have operating costs that are much less correlated with natural gas prices. This increase in the variability of electricty prices - occuring when when natural gas prices are high - is the source of the greater cost savings observed in 2005 and 2008 cases. While the operating cost savings of Table 1 seems significant (between 3 and $10 \%$ ), these must be compared with the purchase costs of the electric heater.

After obtaining a vendor quote, [27], the cost of a 5MW heater - the largest electric heater offered - was estimated to be $\$ 1.1$ million with an installation factor of 3 . Thus, if one assumes a linear Economy-Of-Scale (EOS), then the installed cost of a $35 \mathrm{MW}$ heater would be $\$ 23.1$ million. However, if one assumes the EOS follows the six-tenths rule, [28], then the installed cost would be $\$ 10.6$ million.

Using these capital cost figures, a simple payback period calculation may be performed as capital cost / savings per year. Table 2 indicates the payback period for the three years considered using both types of EOS. Clearly, the payback period calculated from the 2012 data indicates that such a project is unlikely to be implemented. While the payback period figures based on 2005 data seems reasonable, the uncertainty of these figures (possibly increasing by an order of magnitude) indicates the level of risk for such a project.

As one would expect, changes to the maximum furnace turndown, $\delta$, will impact operating cost 
savings. As indicated in Figure 8, the operating cost savings is actually a linear function of $P_{e}{ }^{\max }$, if $P_{e}^{\max } \leq P_{\text {load. }}$ In the case of a linear EOS, this result indicates that payback period will remain constant regardless of $P_{e}{ }^{\max }$. If the EOS follows the 0.6 rule then payback period will decrease with $P_{e}{ }^{\max }$. However, for the case of 2005 , the payback period will reduce only marginally to 2.8 years if $P_{e}^{\max }=P_{\text {load }}$

\section{HEATER WITH ENERGY STORAGE}

In the heater only case, all electric energy purchased would need to be immediately applied to the process. Since the price of electric energy is lower than natural gas for only a portion of the day, the offset of gas usage is restricted to those limited time periods. However, if given an energy storage facility, then purchased electric energy could be applied to the process during other time periods. Using the configuration of Figure 3, energy balances around the furnace and storage unit generate the following constraints.

$$
\begin{aligned}
& \eta_{f} P_{f}(i)+\eta_{s} P_{s}(i)=P_{\text {load }} \\
& E_{s}(i+1)=E_{s}(i)+P_{e}(i)-P_{s}(i)
\end{aligned}
$$

In addition, the physical limits of the equipment can be expressed as:

$$
\begin{aligned}
& 0 \leq P_{s}(i) \leq P_{s}^{\max } \\
& 0 \leq P_{e}(i) \leq P_{e}^{\max } \\
& 0 \leq E_{s}(i) \leq E_{s}^{\text {max }}
\end{aligned}
$$

where $P_{s}^{\max } \hat{=}\left(P_{\text {load }}-\eta_{f} P_{f}^{\min }\right) / \eta_{s}, P_{f}^{\min }$ is as in Section II, and $P_{e}^{\max }$ and $E_{s}^{\max }$ are determined by the size of the electric heater and the storage unit. The optimal operating policy would then be 
determined by the following optimization problem:

$$
\Phi=\min _{P_{f}(i), P_{e}(i), P_{s}(i), E_{s}(i)}\left\{\sum_{i=1}^{M} c_{e}(i) P_{e}(i)+c_{f}(i) P_{f}(i)\right\} \quad \text { s.t. } \quad(8)-(12) i=1 \ldots M
$$

However, in contrast to problem (3), this problem will not have the benefit of a time separation. This is due to equation (9) which possesses variables from multiple time periods. Intuitively, one would expect this to be the case, since the price of electricity several hours into the future will likely influence the actions at the current time.

While it may be computationally tractable to solve the above optimization problem directly, such a procedure is unlikely to be utilized in practice. A common approach to solving similar smart grid coordination problems is the use of Economic Model Predictive Control (EMPC). The procedural starts with the definition of a predictive time index $k$. Then, at each step of actual time, $i$, a predictive model is employed:

$$
\begin{aligned}
& \eta_{f} P_{f}(k \mid i)+\eta_{s} P_{s}(k \mid i)=P_{\text {load }} \\
& E_{s}(k+1 \mid i)=E_{s}(k \mid i)+P_{e}(k \mid i)-P_{s}(k \mid i) \\
& 0 \leq P_{s}(k \mid i) \leq P_{s}^{\max } \\
& 0 \leq P_{e}(k \mid i) \leq P_{e}^{\max } \\
& 0 \leq E_{s}(k \mid i) \leq E_{s}^{\max }
\end{aligned}
$$

where $k=i \ldots i+N-1$. This predictive model is then used within the following optimization problem. 


$$
\begin{array}{r}
\Phi_{i}=\min _{\substack{P_{f}(k \mid i), P_{e}(k \mid i), P_{s}(k \mid i), E_{s}(k \mid i)}}\left\{\sum_{k=1}^{N-1} \hat{c}_{e}(k \mid i) P_{e}(k \mid i)+\hat{c}_{f}(k \mid i) P_{f}(k \mid i)\right\} \\
\text { s.t. }(14)-(18) k=i \ldots i+N-1 \\
E_{s}(i \mid i)=E_{s}(i)
\end{array}
$$

where $E_{s}(i)$ is the actual amount of energy in storage at the actual time $i$. Given the solution to this problem, $P_{f}^{*}(k \mid i), P_{e}^{*}(k \mid i), P_{s}^{*}(k \mid i), E_{s}^{*}(k \mid i) ; k=i \ldots i+N-1$, the actual manipulated variables at time $i$ are set to the first time step of the optimized predictions: $P_{f}(i)=P_{f}^{*}(i \mid i)$, $P_{e}(i)=P_{e}^{*}(i \mid i), P_{s}(i)=P_{s}^{*}(i \mid i)$. At the next time step $i+1$, the updated value of energy in storage $E_{s}(i+1)$ is sent to problem (19), and the process is repeated.

It is important to emphasize that the terms $\hat{c}_{e}(k \mid i)$ and $\hat{c}_{f}(k \mid i)$ are predictions (or forecasts) of the true energy prices. This is because at time $i$ it is impossible to know energy prices $k$ - $i$ time steps into the future. This is the main reason problem (13) would not be used in practice. However for small values of $k-i$, reasonably accurate forecasts can be developed. In the case of natural gas prices, this would correspond to several weeks, possibly months, into the future. In the case of electricity prices, relatively accurate forecasts can be generated $24-48$ hours in advance. An analysis of electricity price forecasting and its impact on EMPC can be found in [14]. However, for the current analysis we will use the assumption of a Full Future Information (FFI) structure, which boils down to knowing all future prices and is reflected as $\hat{c}_{e}(k \mid i)=c_{e}(k)$ and $\hat{c}_{f}(k \mid i)=c_{f}(k)$ for all $k$ and $i$. This assumption will have two impacts. The first is that the closed-loop time-series trajectory resulting from application of the FFI EMPC policy will be nearly identical to that which would result from problem (13), if the horizon, $N$, is sufficiently large. If the horizon is insufficiently large, then the EMPC policy will suffer from inventory creep, see [21] for details. 
The second impact is that the operating costs resulting from application of this FFI EMPC will provide a lower bound to the actual operating costs that would result if one were to implement EMPC without perfect forecasting. It should also be noted that if the assumption of $P_{\text {load }}$ being a constant was removed (i.e., $P_{\text {load }}$ is assumed a disturbance), then forecasts, $\hat{P}_{\text {load }}(k \mid i)$, would also need to be generated, the quality of which would also impact closed-loop EMPC performance.

Example 2: Assume $P_{\text {load }}=87.9 \mathrm{MW}, \eta_{f}=0.75, \eta_{s}=0.98, \delta=39 \%\left(\right.$ which makes $P_{s}^{\max }=35 \mathrm{MW}$ ), $P_{e}{ }^{\max }=70 \mathrm{MW}, E_{s}^{\max }=200 \mathrm{MWh}$ and $N=24$. In this case, an example of the closed-loop FFI EMPC trajectory is depicted in Figure 9. The first point to note is that $P_{f}(i)$ is equal to $P_{f}{ }^{\text {min }}$ for large periods (days 287-289) and the periods in which $P_{f}(i) \geq P_{f}{ }^{\text {min }}$ correspond to large intervals of $c_{R}(i) \geq 0$ (for example 285.25-286 and 286.25-287). The second observation is that for the most part $P_{e}(i)$ takes only three values $(0,35$ and $70 \mathrm{MW})$ and has a response similar to the heater only case: $P_{e}(i)=P_{e}^{\max }$ if $c_{R}(i)<0$ and $P_{e}(i)=0$ if $c_{R}(i) \geq 0$. The $P_{e}(i)=35 \mathrm{MW}$ cases result when $c_{R}(i)<0$ and the controller is waiting for a lower price of electricity, forecasted to occur a few hours into the future. Examples of this can be found just before 286 and just before 288. A similar phenomenon occurs when $c_{R}(i)<0$ for long periods. For example, during day 288 the EMPC starts with $P_{e}(i)=70 \mathrm{MW}$, to capture low electric prices, but then switches to $P_{e}(i)=$ $35 \mathrm{MW}$ when the price rises, even though $c_{R}(i)$ is still $<0$. This is due to the fact that the storage unit is at its maximum capacity. Then, when prices go through a few small hills and valleys, $P_{e}(i)$ is cycled to capture the best prices. As one might expect, increasing the size of the storage unit will reduce this type of behavior by allowing the controller to store energy during more of the $c_{R}(i)<0$ 
periods, which will lower operating costs by having $P_{f}=P_{f}{ }^{\text {min }}$ for longer intervals.

Table 3 shows the operating cost savings using data from the three years. While each has a savings that is about $50 \%$ higher than the heater only case, it is important to remember that the current case requires significantly more equipment - in addition to the storage facility, the current example uses an electric heater that is twice the size of that in Example 1. Estimates of the capital costs associated with a molten salt energy storage facility can be found in $[29,30]$. However, rather than consider that level of detail, a back-of-the-envelope type calculation is given in Table 4 , where only the cost of the heater is considered (i.e., the cost of the storage unit is assumed zero). This simpler calculation will provide a lower bound on payback period. In the case of linear EOS, all of the payback periods increase as compared to Example 1. This should be a bit surprising given the assumption of zero capital costs for the storage unit. However, the fact that the operating cost savings increased by roughly $50 \%$, while the size of the electric heater was doubled should indicate the source of this result. In the case of the EOS following 0.6 rule, the payback period is about the same or slightly better than Example 1. However, if Example 1 were recalculated using a 70MW heater then the payback period for Example 2 would be higher for all years.

The source of these somewhat puzzling results may be that the size of the storage facility is non-optimal. Figure 10 illustrates operating cost savings as a function of storage size, for all values of $P_{e}^{\max }$ and compares with the scenario of Example 1. The first point to note is that if $P_{e}^{\max }$ is less than $35 \mathrm{MW}$, then the saving is identical for all cases. This is due to the fact that $P_{s}^{\max }$ is set to 35MW. Thus, if $P_{e}^{\max } \leq P_{s}^{\max }$, then the optimal policy is essentially the same as in the heater only 
case. That is, if $c_{R}(i)<0$ set $P_{e}=P_{e}^{\max }$ and bypass the storage by sending all of this heat directly to the process. If $P_{e}^{\max }>P_{s}^{\max }$, then there is an opportunity to purchase more electric energy that can be immediately used by the process, which can be stored and used at a later time. However, this benefit is limited by the capacity of the storage facility. For example, in the 50MWh storage case there is almost no benefit to increasing $P_{e}^{\max }$ beyond $35 \mathrm{MW}$. In contrast, the savings could be doubled if given an $800 \mathrm{MWh}$ storage facility and a sufficiently large heater.

While it may appear that the without storage curve provides an upper bound to possible savings, consider the case of $P_{s}^{\max }=70 \mathrm{MW}$, depicted in Figure 11 and representing $\delta=79 \%$. In this case, cost saving above the heater only case is easily obtained for sufficiently large values of $E_{s}^{\max }$ and $P_{e}^{\max }$. It is finally noted that if $P_{s}^{\max }$ is sufficiently large, then one would find a result aligned with initial intuition. That is, the inclusion of a storage facility will have an operating cost savings that is bounded below by the heater only case (see Figure 12).

The above disconnect with intuition seems to stem from the fact that our system has two energy sources. This is in contrast with other smart grid coordinated systems - for example in a building cooling system, [11-14] - where electricity is the only energy source. In these systems, one may use time-shifting arguments to conclude that the addition of storage will always reduce operating costs with respect to the baseline. Of course, in those cases the baseline is likely higher since the system is limited to only one energy source. 


\section{CONCLUSIONS}

In this work, we have explored a particular smart grid coordination opportunity that is suitable for use in chemical plants. The first major conclusion is that the operating cost savings is heavily influenced by the amount of variability found in the price of electricity. Furthermore, we have anecdotally observed that electricity price variability is correlated with natural gas price. The second major conclusion is that while operating cost savings may be significant, these savings will outweigh equipment costs only under the most favorable conditions. Finally, it was observed that the inclusion of an energy storage unit did little to improve the economics of the process, even under the assumption of zero capital costs for the storage equipment. The source of this non-intuitive result was illustrated to be due to the fact that two energy sources were available, one of which has a maximum turndown factor less than 1 .

While the presented results may appear discouraging, it is important to remember that the scope of the present analysis was limited to the economic response aspect of demand response. Future work will investigate the proposed configuration in the context of ancillary services, also known as contingency response and regulation response (see [8] for details). In both types of demand response, a distinct revenue stream can be received by turning over control of electric power usage to the power system operator. Since the proposed configuration will allow for frequent changes in electric power consumption with virtually no change in the temperature of the hot oil stream (due to the response of the furnace control system), providing such services will have almost no impact to the production side of the chemical plant. 


\section{ACKNOWLEDGEMENTS}

Financial support from the National Science Foundation (CBET-0967906) is gratefully acknowledged.

\section{REFERENCES}

[1] Farhangi, H. (2010) The path of the smart grid, IEEE Power and Energy Magazine, 8(1), pp. $18-28$

[2] Ipakchi, A., F. Albuyeh (2009) Grid of the future, IEEE Power and Energy Magazine, 7(2), pp. $52-62$

[3] Grainger, J. J., W.D. Stevenson (1994) Power system analysis, New York, NY: McGraw-Hill, Inc.

[4] Pennsylvania, New Jersey, Maryland Interconnection (2013) Real-time historical data: http://www.pjm.com/markets-and-operations/energy/real-time/historical-bid-data.aspx (accessed on Jun 25, 2013)

[5] Lindenberg, S., Smith, B., O’Dell, K., DeMeo, E., Ram, B. (2008) 20\% Wind Energy by 2030: Increasing Wind Energy's Contribution to U.S. Electric Supply. DOE/GO-102008-2567

[6] Rahimi, F., A. Ipakchi (2010) Demand response as a market resource under the smart grid paradigm, IEEE Trans. Smart Grid, 1(1), pp.82-88 
[7] Walawalkar, R., S. Fernands, N. Thakur, K.R. Chevva (2010) Evolution and current status of demand response (DR) in electricity markets: Insights from PJM and NYISO, Energy, 35(4), pp 1553-1560

[8] Chmielewski, D.J. (2014) Special Section - Energy: Smart Grid: The Basics — What? Why? Who? How?, CEP Magazine, August 2014, pp 28-34

[9] Chen, Z., L. Wu, Y. Fu (2012) Real-time price-based demand response management for residential appliances via stochastic optimization and robust optimization, IEEE Trans. Smart Grid, 3(4), pp.1822,1831

[10] O'Neill, D., M. Levorato, A. Goldsmith, U. Mitra (2010) Residential demand response using reinforcement learning, In Proc IEEE Int. Conf. Smart Grid Comm. pp. 409-414, Gaithersburg, MD, October 2010

[11] Halvgaard, R., N. K. Poulsen, H. Madsen, and J. B. Jørgensen (2012) Economic model predictive control for building climate control in a smart grid. In Proc 2012 Innovative Smart Grid Tech, Washinton D.C., 2012

[12] Ma, J., J. Qin, T. Salsbury, P. Xu (2012) Demand reduction in building energy systems based on economic model predictive control, Chem. Eng. Sci., 67(1), pp 92-100

[13] Oldewurtel, F., A. Ulbig, A. Parisio, G. Andersson, M. Morari (2010) Reducing peak electricity demand in building climate control using real-time pricing and model predictive control, In Proc IEEE Conf. Dec. Contr., pp.1927,1932, Atlanta, GA, December 2010

[14] Mendoza-Serrano, D.I, D.J. Chmielewski (2014) Smart grid coordination in building HVAC systems: EMPC and the impact of forecasting, J. Proc Contr., 24, pp 1301-1310 
[15] Todd, D., B. Helms, M. Caufield, M. Starke, B. Kirby, J. Kueck (2009) Providing Reliability Services through Demand Response: A Preliminary Evaluation of the Demand Response Capabilities of Alcoa Inc., DOE Report: ORNL/TM-2008/233

[16] Castro, P.M., L. Sun, I Harjunkoski (2013) Resource-task network formulations for industrial demand side management of a steel plant, Ind Eng. Chem. Res., 52, pp. $13046-13058$

[17] Huang, R., E. Harinath, L.T. Biegler (2011) Lyapunov stability of economically oriented NMPC for cyclic processes, J Proc. Contr., 21, pp 501-509

[18] Baumrucker, B.T., L.T. Beigler (2010) MPEC strategies for cost optimization of pipeline operations, Comp. Chem. Eng., 34, pp 900-913

[19] Mitra, S. I.E. Grossmann, J.M. Ponto, N. Arora (2012) Optimal production planning under time-sensitive electricity prices for continuous power-intensive processes, Comp. Chem. Eng., 38, pp 171-184

[20] Mendoza-Serrano, D. I., D. J. Chmielewski (2013) Demand response for chemical manufacturing using economic MPC. In Proc. 2013 Am. Contr. Conf., pp 6655-6660, Washington, DC, June 2013

[21] Omell B.P., D. J. Chmielewski (2013) IGCC power plant dispatch using infinite-horizon economic model predictive control. Ind, Eng, Chem, Res, 52(9), pp 3151-3164

[22] Rawlings, J.B., D. Angeli, C. N. Bates (2012) Fundamentals of economic model predictive control, In Proc IEEE Conf. Dec. Contr., pp 3851-3861, Maui, HI, December 2012 
[23] Ellis, M., H. Durand, P.D. Christofides (2014) A tutorial review of economic model predictive control methods, J. Proc. Contr., 24(8), pp 1156-1178

[24] Trana, T., K-V. Linga, J.M. Maciejowski (2014) Economic Model Predictive Control - A Review, In Proc 31st Int. Symp. Aut. and Rob in Construction and Mining

[25] Adeodu, O., D. J. Chmielewski (2013) Control of electric power transmission networks with massive energy storage using economic MPC. In Proc. 2013 Am. Contr. Conf., pp 5839-5844, Washington, DC, June 2013

[26] Energy Information Agency (2013) Historical nature gas industrial price data : http://www.eia.gov/dnav/ng/hist/n3035us3m.htm (accessed on Jun 30, 2013)

[27] O’Brien, D. (2013) Personal communication with Armstrong-Chemtech Group, West Chester, PA (www.rmarmstrong.com)

[28] Seider, W.D., J.D. Seader, D.R. Lewin (1999) Product \& process design principles: Synthisis, analysis, and evaluation $2^{\text {nd }}$ Ed., Wiley \& Sons: New York, NY

[29] Pacheco, J., S. Showalter, W. Kolb (2002) Development of a molten-salt thermocline thermal storage system for parabolic trough plants, Trans ASME. J. Solar Energy Eng., 124(2), pp. 153-159

[30] Herrmann, U., D. Kearney (2002) Survey of thermal energy storage for parabolic trough power plants, Trans ASME. J. Solar Energy Eng., 124(2), pp. 145-152

[31] Zachar, M; M. Trifkovic; P. Daoutidis, Policy effects on microgrid economics, technology selection, and environmental impact, Comp. \& Chem. Eng., in review 
[32] Yang, W-M.; B.P. Omell; D.J. Chmielewski (2012) Controller design for dispatch of IGCC power plants, In Proc. 2012 Am. Contr. Conf., pp 4281-4286, Montreal, Canada, June 2012

[33] Salsbury, T.; P. Mhaskar; S.J. Qin (2013) Predictive control methods to improve energy efficiency and reduce demand in buildings, Comp. \& Chem. Eng., 51(5) pp 77-85

[34] Touretzky, C.R.; M. Baldea (2014) Integrating scheduling and control for economic MPC of buildings with energy storage, J. Proc. Cont., 24(8), pp 1292-1300. 


\section{Tables}

Table 1: Annual operating cost (in million) for Example 1

\begin{tabular}{lccc}
\hline Year & 2005 & 2008 & 2012 \\
\hline Baseline (without electric heater) & $\$ 34.6$ & $\$ 39.2$ & $\$ 19.7$ \\
Example 1 (with electric heater) & $\$ 32.0$ & $\$ 36.1$ & $\$ 19.2$ \\
Operating cost savings & $\$ 2.6$ & $\$ 3.1$ & $\$ 0.5$ \\
Percent of baseline & $7.5 \%$ & $7.9 \%$ & $2.5 \%$ \\
\hline
\end{tabular}


Table 2 : Payback periods for Example 1

\begin{tabular}{lccc}
\hline Year & 2005 & 2008 & 2012 \\
\hline Operating cost savings (millions) & $\$ 2.6$ & $\$ 3.1$ & $\$ 0.5$ \\
Payback period with linear EOS (years) & 8.9 & 7.4 & 46 \\
\begin{tabular}{l} 
Payback period with 0.6 rule EOS (years) \\
\hline
\end{tabular} & 4.1 & 3.4 & 21 \\
\hline
\end{tabular}


Table 3: Annual operating costs (in million) for Example 2

\begin{tabular}{lccc}
\hline Year & 2005 & 2008 & 2012 \\
\hline Baseline (without electric heater or storage) & $\$ 34.6$ & $\$ 39.2$ & $\$ 19.7$ \\
Example 2 (with electric heater and storage) & $\$ 30.6$ & $\$ 34.3$ & $\$ 18.7$ \\
Operating cost savings & $\$ 4.0$ & $\$ 4.9$ & $\$ 1.0$ \\
Percent of baseline & $11.6 \%$ & $12.5 \%$ & $5.1 \%$ \\
\hline
\end{tabular}


Table 4: Payback period for Example 2

\begin{tabular}{lccc}
\hline Year & 2005 & 2008 & 2012 \\
\hline Operation cost savings (millions) & $\$ 4.0$ & $\$ 4.9$ & $\$ 1.0$ \\
70 MW heater cost with linear EOS (millions) & $\$ 46.2$ & $\$ 46.2$ & $\$ 46.2$ \\
Payback period with linear EOS (years) & 11.5 & 9.4 & 46 \\
70 MW heater cost with 0.6 rule EOS (millions) & $\$ 16.1$ & $\$ 16.1$ & $\$ 16.1$ \\
Payback period with 0.6 rule EOS (years) & 4.0 & 3.3 & 16 \\
\hline
\end{tabular}




\section{Figures}

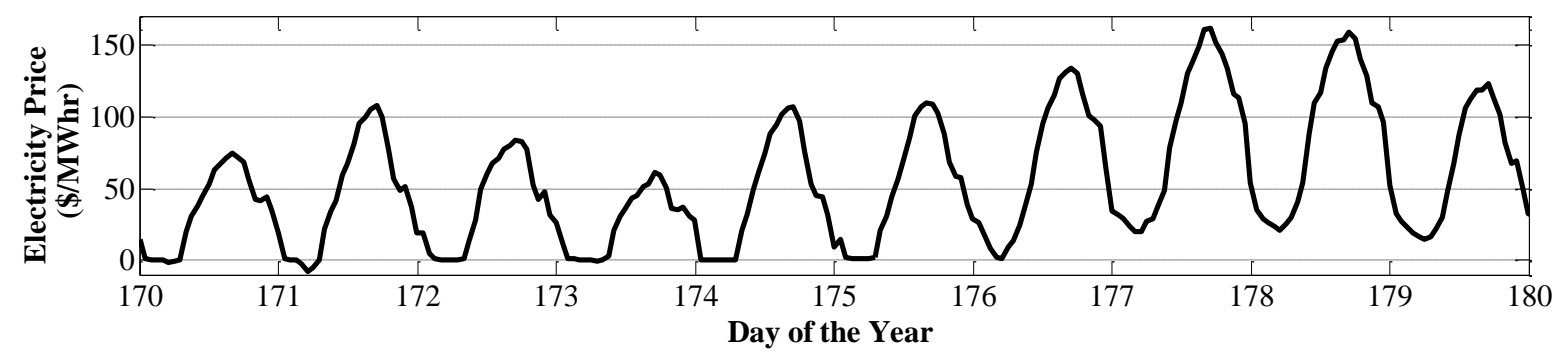

Figure 1: Historic electricity prices (Chicago, 2008), [4] 


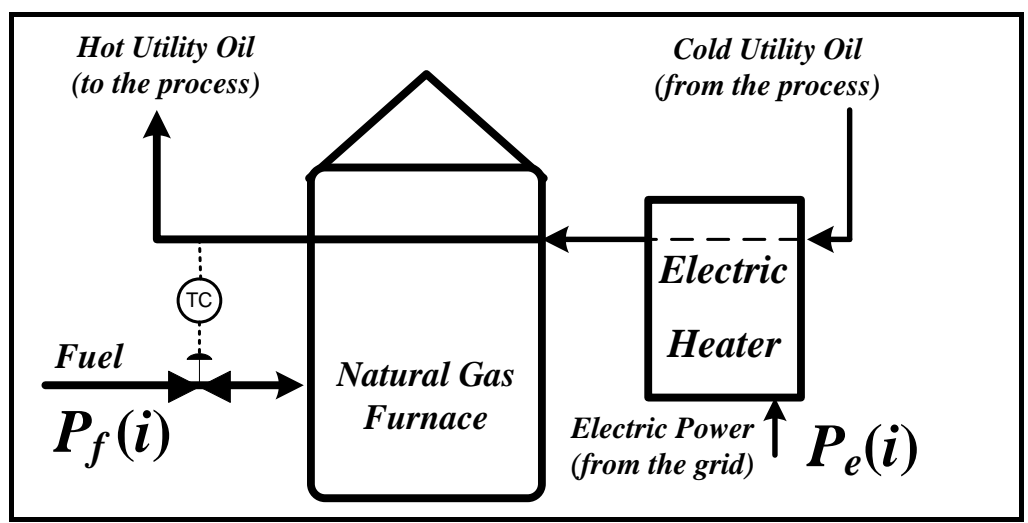

Figure 2: Simplified process diagram of a utility plant with electric heating. 


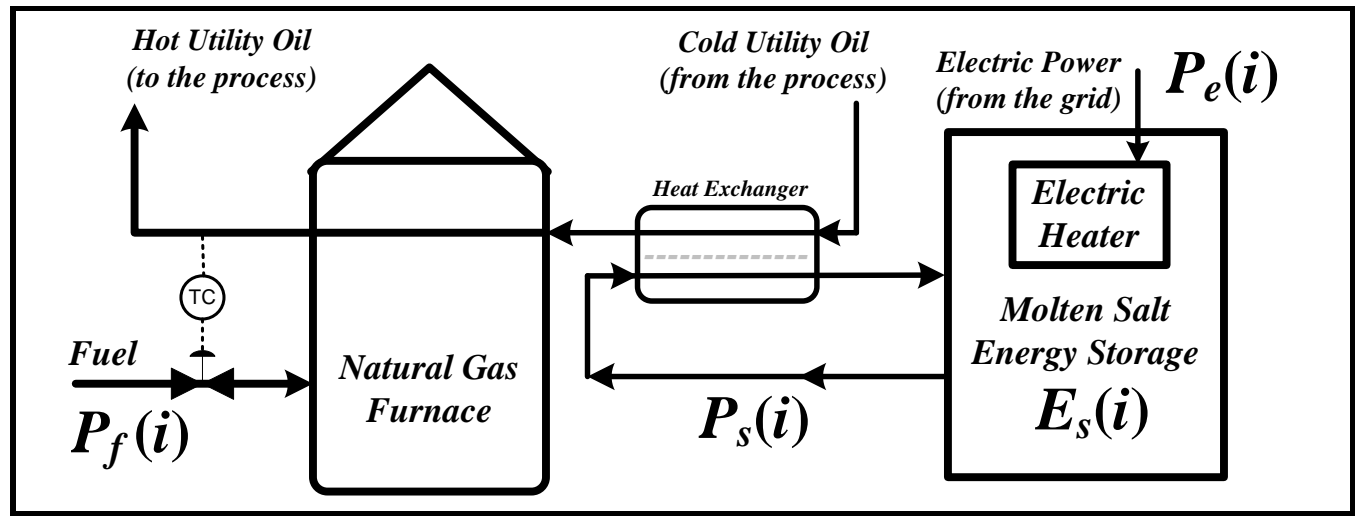

Figure 3: Simplified process diagram of a utility plant with electric heating and energy storage. 

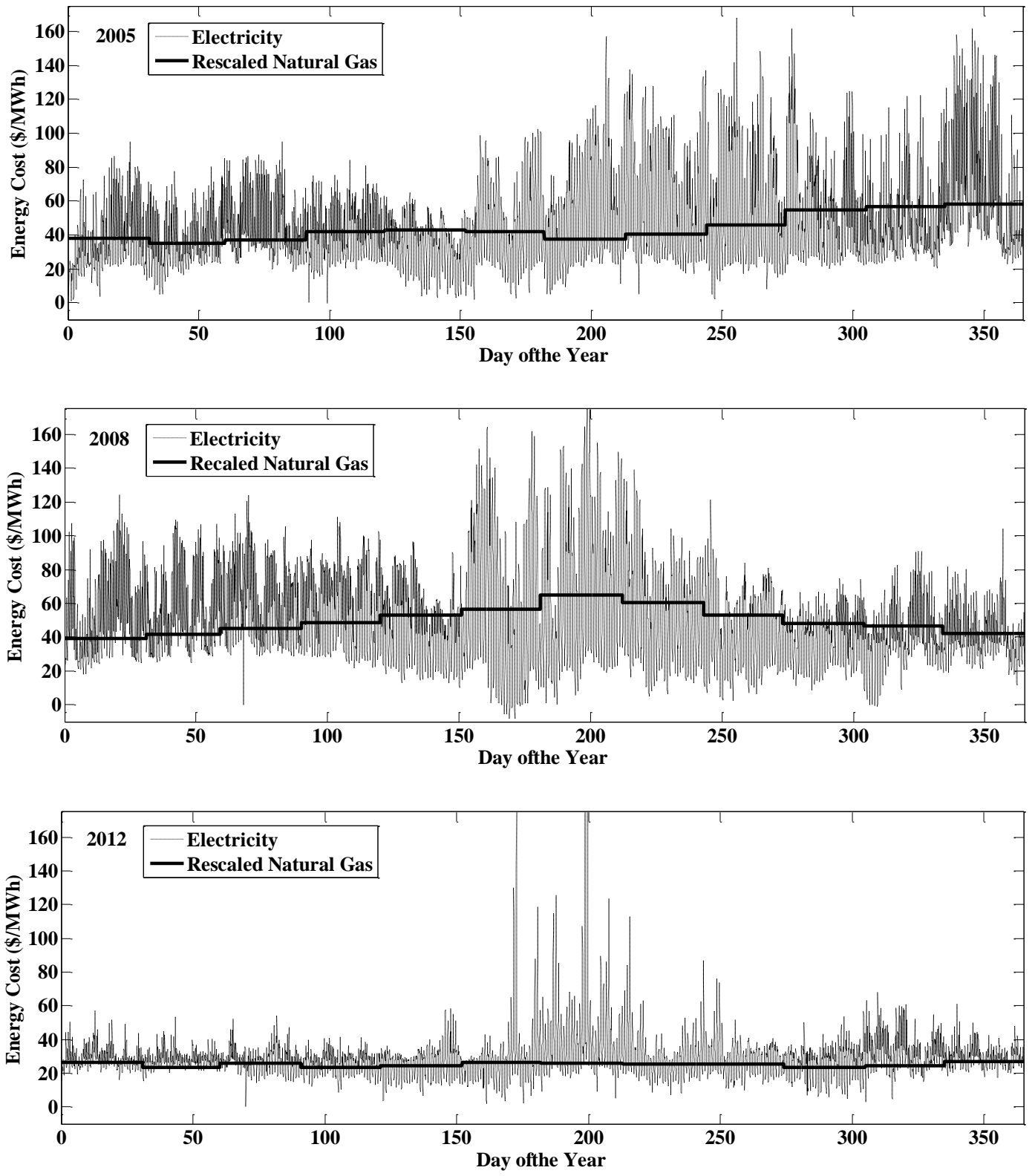

Figure 4: Price of electricity in Chicago HUB [4] and rescaled price of natural gas [26]. 

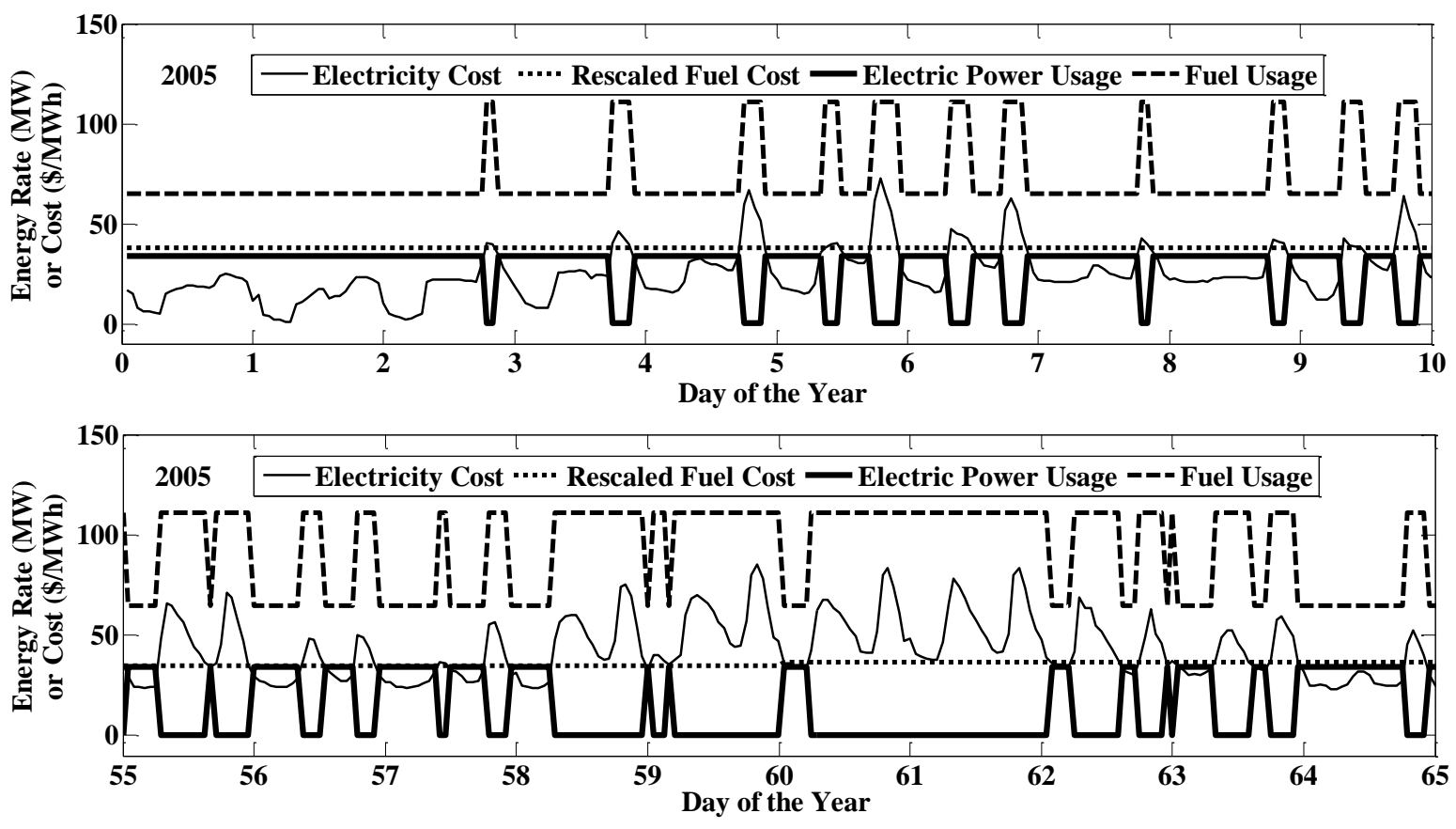

Figure 5: Time-series of optimal operating policies for Example 1 during 2005. 

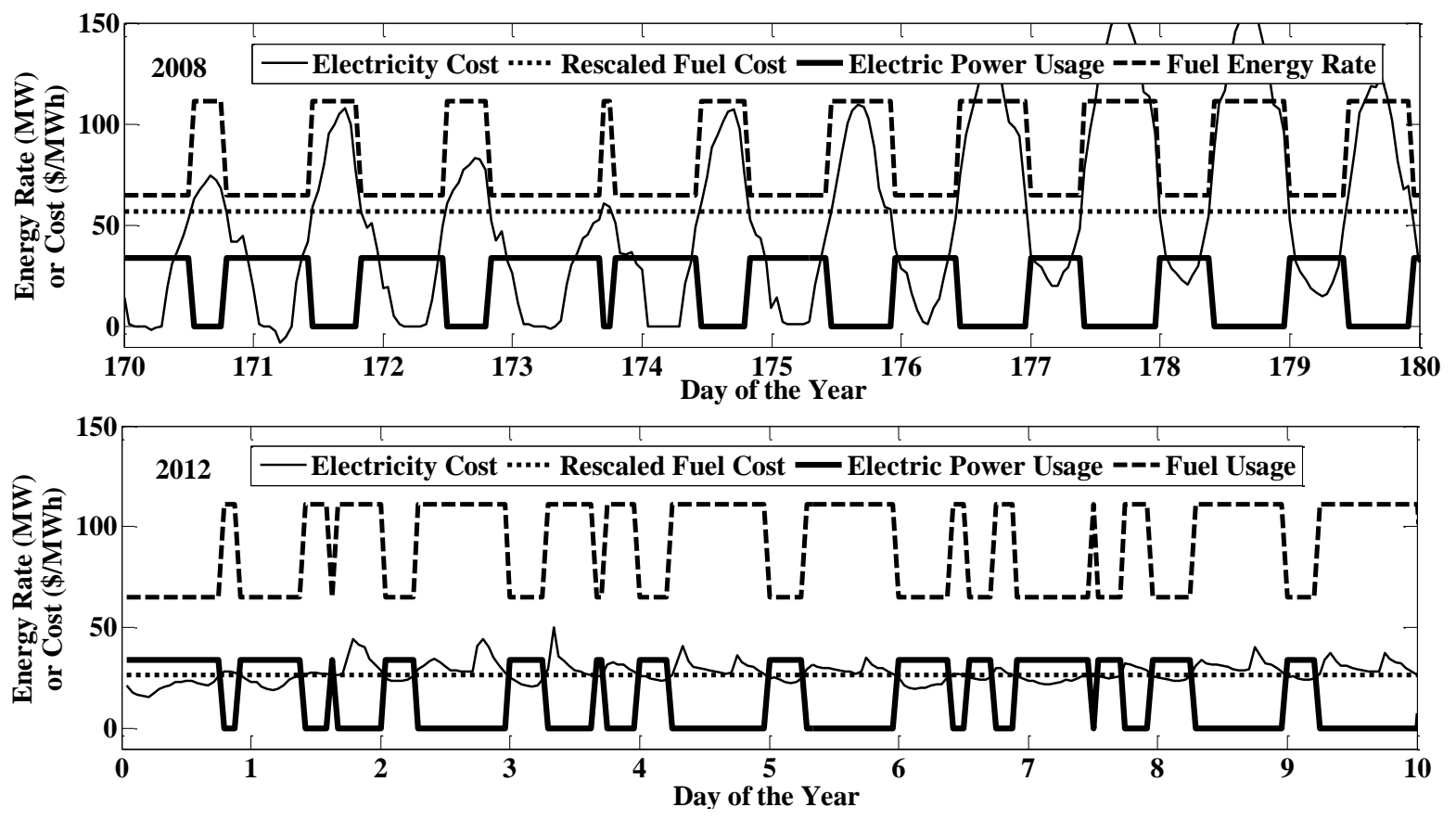

Figure 6: Time-series of optimal operating policies for Example 1 during 2008 (top) and 2012 (bottem). 


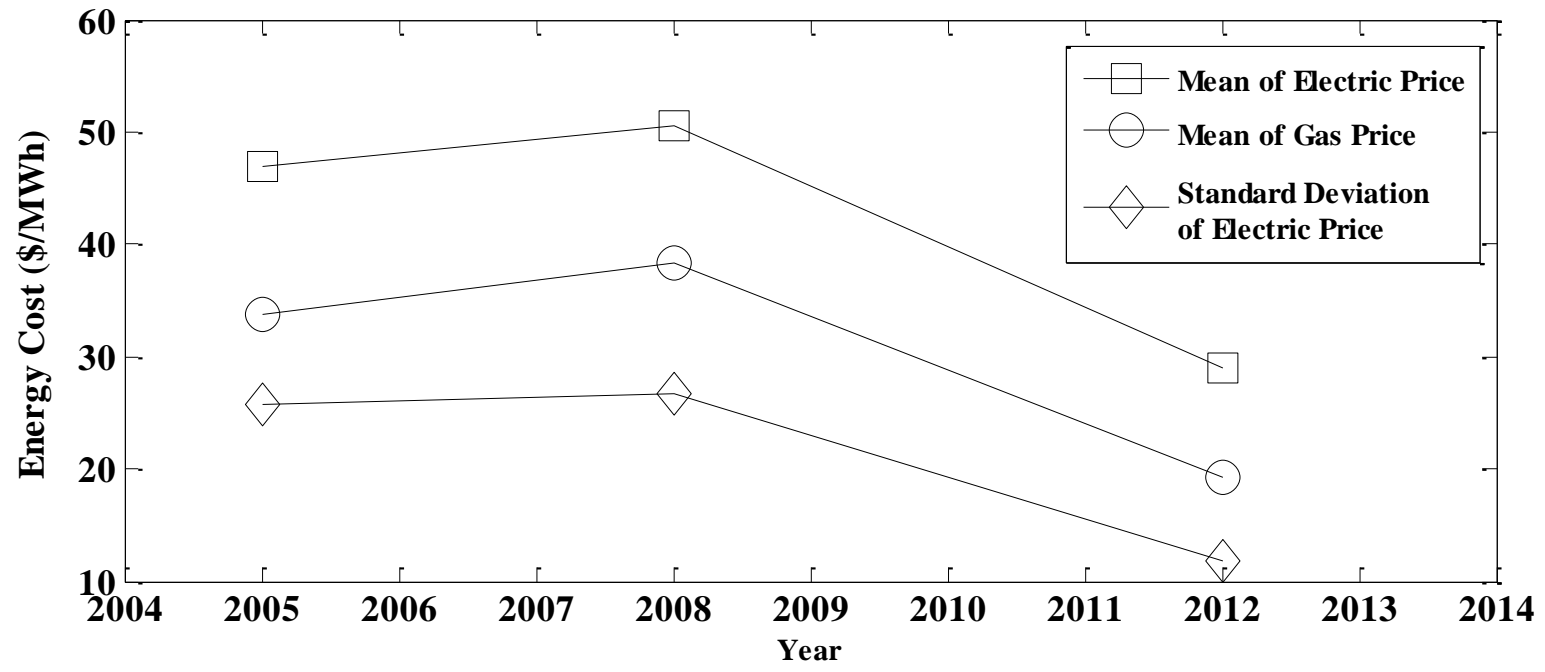

Figure 7: Correlation between Average Energy Costs and Variability of Electricity Costs. 


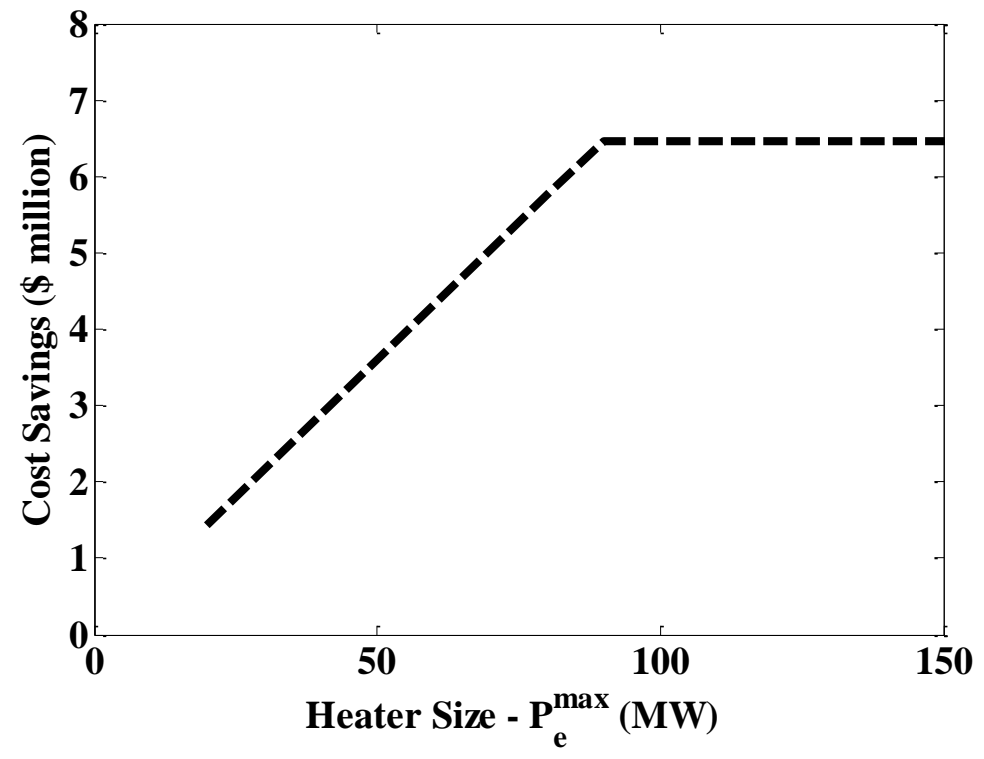

Figure 8: Operating cost saving vs. electric heater size for Example 1 (based on 2005 data) 

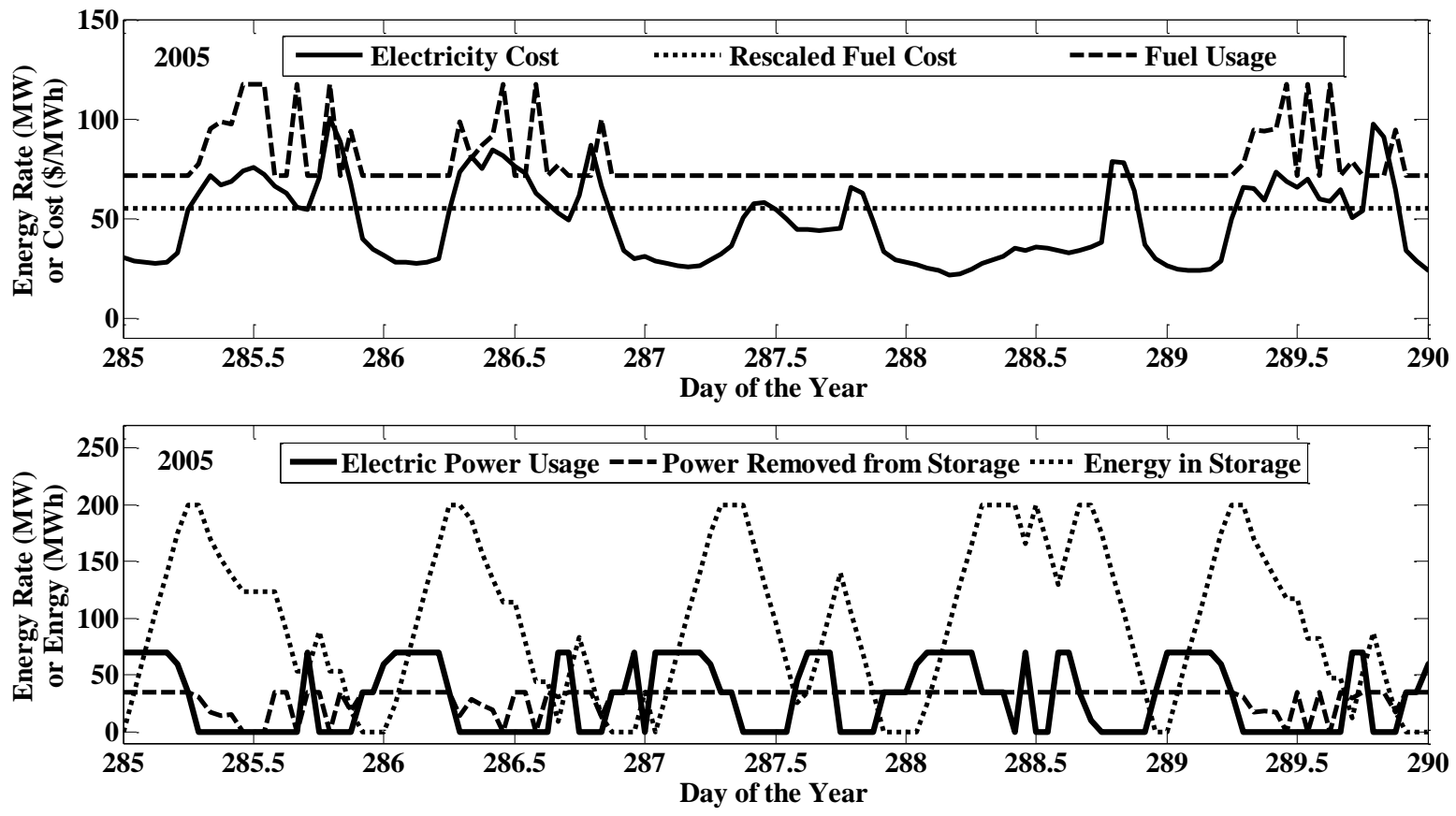

Figure 9: Time-series of optimal operating policies for Example 2 during 2005. 


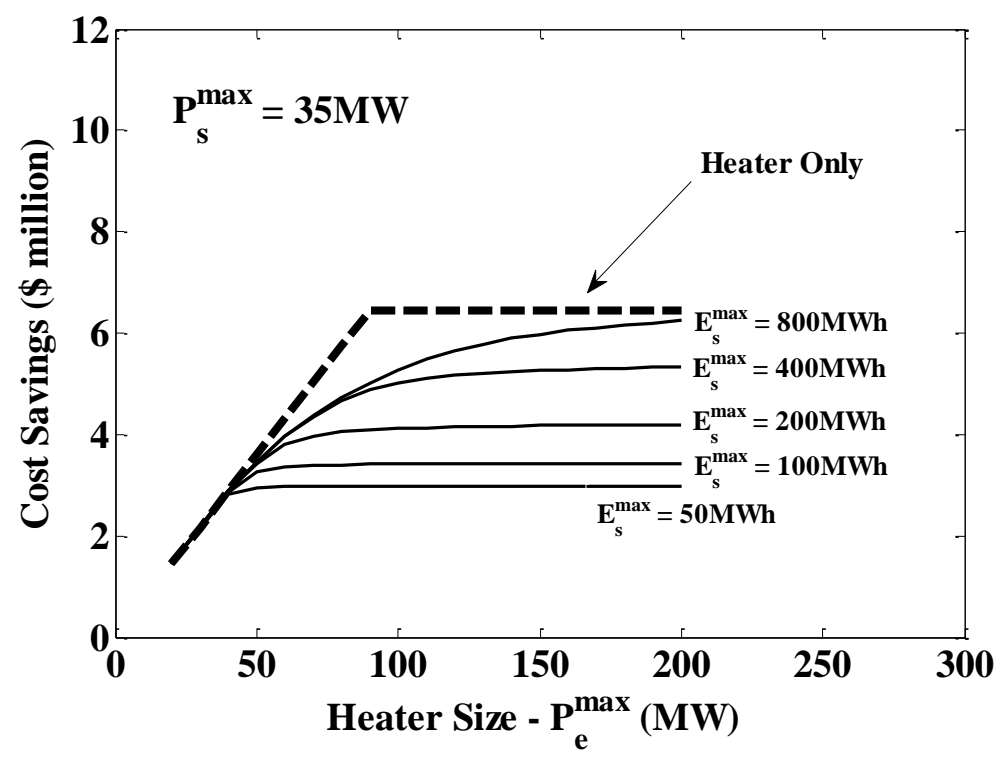

Figure 10: Cost Savings vs. Heater Size $\left(P_{s}^{\max }=35 \mathrm{MW}, 2005\right.$ data $)$ 


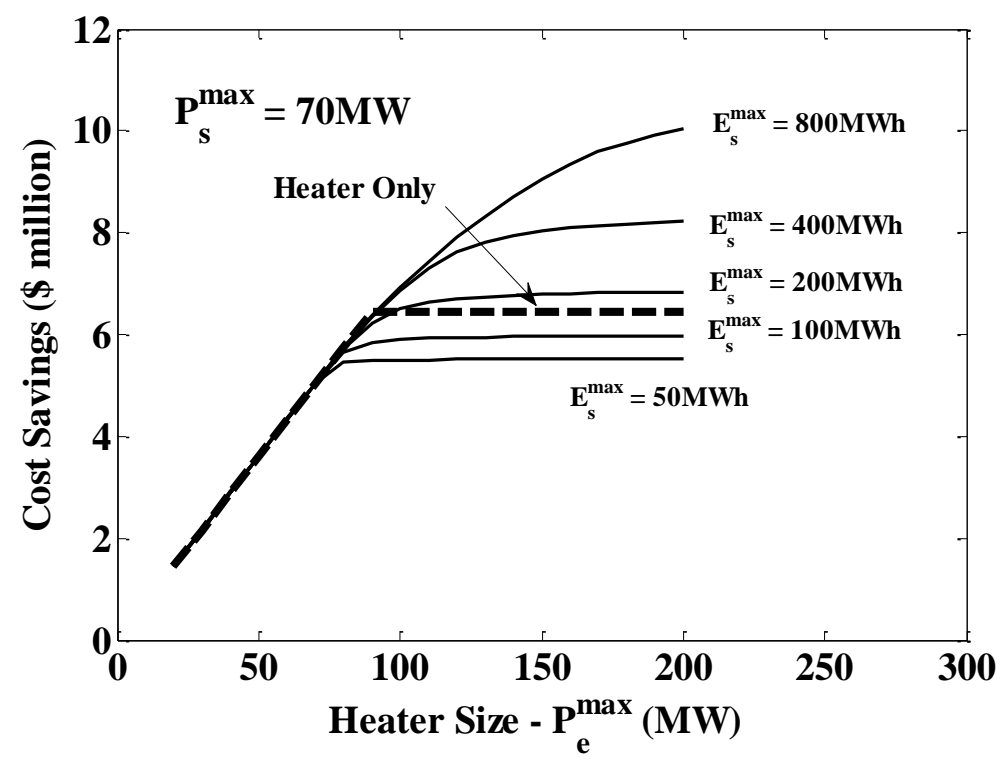

Figure 11: Cost Savings vs. Heater Size ( $P_{s}^{\max }=70 \mathrm{MW}, 2005$ data) 


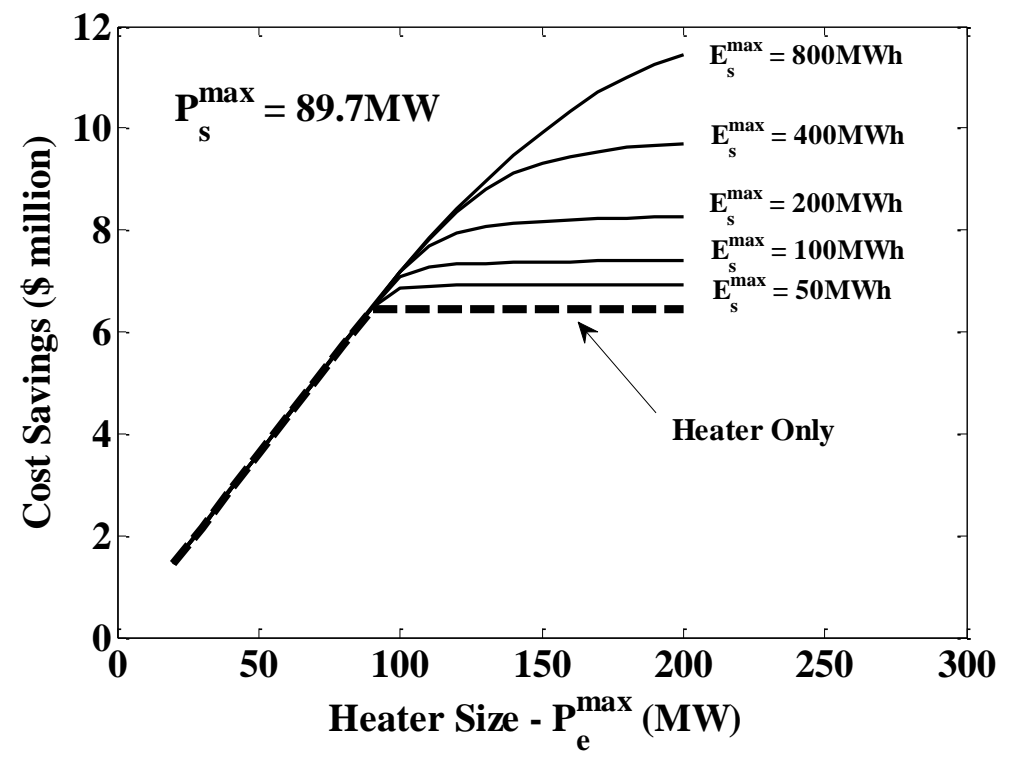

Figure 12: Cost Savings vs. Heater Size ( $P_{s}^{\max }=89.7 \mathrm{MW}, 2005$ data) 